\title{
Quadrupling of unit cell in half-filled domain walls in the cuprates
}

\author{
Andrzej M. Oleś ${ }^{a, b, *}$, Jan Zaanen ${ }^{c}$ \\ a Institute of Physics, Jagellonian University, Reymonta 4, PL-30059 Kraków, Poland \\ ${ }^{\mathrm{b}}$ Max-Planck-Institut FKF, Heisenbergstr. 1, D-70569 Stuttgart, Germany \\ ${ }^{c}$ Institute Lorentz, Leiden University, POB 9506, NL-2300 RA Leiden, Netherlands
}

\begin{abstract}
We investigate the stability of the domain walls in the stripe phase of the cuprates filled by one hole per two unit cells by semiclassical calculations using the extended Hubbard model with electron-phonon coupling. The walls are locally stable due to quadrupling of unit cell along the wall which involves the modulation of coupled spin and charge density. Such states could be measured by neutron spectroscopy.
\end{abstract}

Keywords: Stripe phase; Domain wall; Hubbard model; Cuprates

When holes are doped into a two-dimensional (2D) antiferromagnet, a number of different symmetrybroken states may occur. One of them is the so-called stripe phase characterized by simultaneous ordering of spins and charges. The stripe phase instability was predicted within the semiclassical calculations for the Hubbard model $[1,2]$ and confirmed also in the multiband model [3]. This phase with the filling of one hole per one domain wall (DW) unit cell, called below filled domain wall (FDW), has been first observed in the nickelates [4]. There are indications that charge ordering occurs as well in manganese oxides which show collosal magnetoresistence [5]. Thus, the physical understanding of mechanisms of such instabilities and the quantum fluctuations in the stripe phase is of great physical interest $[6,7]$.

The stripe phase has also been observed recently in $\mathrm{La}_{1.6-x} \mathrm{Nd}_{0.4} \mathrm{Sr}_{x} \mathrm{CuO}_{4}$ [8]. In contrast to the nickelate compound $\mathrm{La}_{2-x} \mathrm{Sr}_{x} \mathrm{CuO}_{4}$, where the DWs are diagonal [4], one finds here horizontal DWs which can be explained by a weaker Coulomb interaction $U$ in

\footnotetext{
* Corresponding author.
}

the cuprate $[1,2]$. More importantly, the superconducting compound becomes insulating at the doping $\delta=\frac{1}{8}$ which shows that the filling is only one hole per two domain wall unit cells. It is the motivation of the present contribution to reveal the reasons of stability of the half-filled domain wall (HFDW) using the simplest realistic approach, the self-consistent Hartree-Fock approximation.

The multiband models for the cuprates can be mapped on the effective one-band Hamiltonian [9]. Hence, we adopt the point of view that the physical properties of the cuprates are faithfully represented by the effective extended Hubbard model on a 2D square lattice,

$$
\begin{aligned}
H= & \sum_{i, \delta, \sigma} t_{0}\left(1+\alpha u_{i \delta}\right) c_{i \sigma}^{\dagger} c_{i+\delta, \sigma}+U \sum_{i} n_{i \uparrow} n_{i \downarrow} \\
& +V \sum_{i, \delta, \sigma, \sigma^{\prime}} n_{i \sigma} n_{i+\delta, \sigma^{\prime}}+\frac{1}{2} K \sum_{i, \delta} u_{i, \delta}^{2} .
\end{aligned}
$$

The notation is standard, and the Coulomb interactions are represented by a large on-site term $U$, and a smaller element $V$. The electrons couple to the lattice by a 
Peierls term $(\sim \alpha)$, with $u_{i \delta}$ standing for the change of the $(i, i+\delta)$ bond. Thus, in the neighbourhood of halffilling the kinetic energy is given by the renormalized hopping,

$t=t_{0}\left[1+\alpha u_{i \delta}(n=1)\right]$,

with $t<t_{0}$. It defines the electron-phonon coupling constant, $\lambda=\alpha^{2} t / K$.

We performed a standard Hartree-Fock approximation which includes both longitudinal and transverse spin components, as for instance,

$$
\begin{aligned}
n_{i \uparrow} n_{i \downarrow} \approx & n_{i \uparrow}\left\langle n_{i \downarrow}\right\rangle+\left\langle n_{i \uparrow}\right\rangle n_{i \downarrow}-\left\langle S_{i}^{+}\right\rangle S_{i}^{-}-\left\langle S_{i}^{-}\right\rangle S_{i}^{+} \\
& -\left\langle n_{i \uparrow}\right\rangle\left\langle n_{i \downarrow}\right\rangle+\left\langle S_{i}^{+}\right\rangle\left\langle S_{i}^{-}\right\rangle,
\end{aligned}
$$

where $S_{i}^{+}=c_{i \uparrow}^{\dagger} c_{i \downarrow}$. This results in a one-particle problem to be solved self-consistently with the local fields $\phi_{i z} \equiv\left\langle n_{i \uparrow}-n_{i \downarrow}\right\rangle / 2, \phi_{i \pm} \equiv\left\langle S_{i}^{ \pm}\right\rangle$, and $u_{i \delta}$. It is crucial to include the equilibrium relation between the bond length and the bond order,

$u_{i \delta}=-\frac{\alpha t_{0}}{K} \sum_{\sigma}\left\langle c_{i \sigma}^{\dagger} c_{i+\delta, \sigma}\right\rangle$

We minimized the ground state with respect to spin rotations in the $(x, z)$ plane.

At large values of $U / t \simeq 4$ one finds that the doped holes change the density only on the (vertical) wall itself and on the sites next to it. This motivates an analytic solution of a three-site problem which shows that the stability of a DW follows then from the binding energy of two electrons with opposite spins across the DW [10]. Naively this suggests that the FDW should be more stable than the half-filled one.

We have investigated the HFDWs stability by comparing the energies of self-consistent solutions of 2D $N=N_{x} \times N_{y}$ supercells, being even times even for the cases with either zero or two walls, and odd times even for those with one DW. The energy gains normalized per one excess hole,

$E=\frac{E_{0}-E_{\mathrm{AF}}}{N_{\mathrm{h}}}+4 V$,

defined with respect to the energy of the undoped antiferromagnetic lattice, with $E_{\mathrm{AF}}=N \varepsilon_{\mathrm{AF}}$, are an absolute measure of stability of various solutions. The

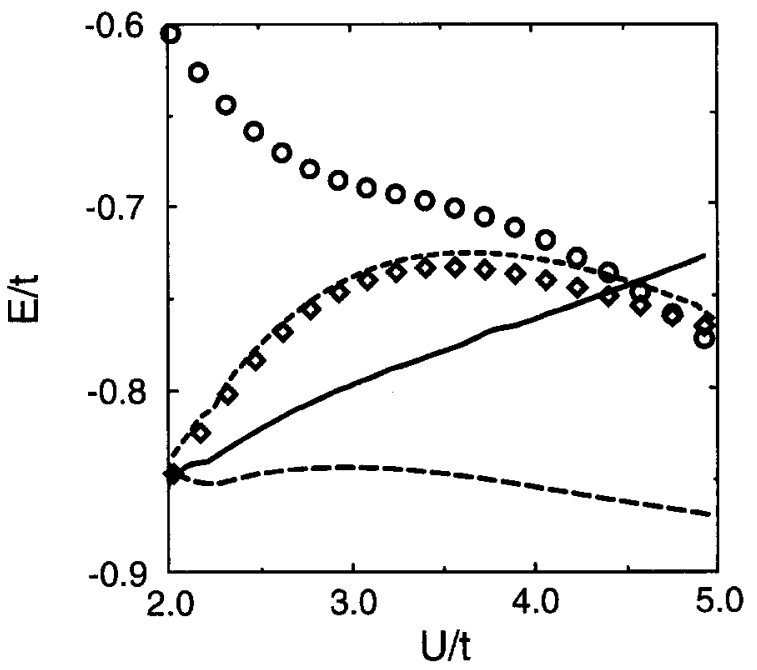

Fig. 1. The energies per doped hole in various states as function of $U / t$ for $\lambda=0.5$, and $V=0.25|t|$. A hole in a HFDW is shown by short dashed line and diamonds for the on-wall SDW and CDW, respectively; in a FDW by long-dashed line, while circles and full line stand for isolated polarons and a spiral.

gain of the intersite Coulomb energy $\sim V$ has been compensated by the term $4 \mathrm{~V}$ for convenience. This study allows us to conclude that the HFDWs represent locally stable structures, but are always less stable than the FDWs, independently of the actual values of $V$ and $\lambda$, also for $V<0$. A typical result is shown in Fig. 1 . We note that the coupling to the lattice increases somewhat the binding energy (5) of a hole in a HFDW, but does not suffice to stabilize it with respect to a hole in a FDW. Moreover, even the spiral is more stable in a broad range of intermediate values of $2<U / t<4.4$.

The main result of the semiclassical calculations [10] is a qualitative criterion for the local stability of HFDWs in the cuprates. One finds that the period on the stripes should become four times the lattice constant, due to either a new spin-density wave (SDW, shown in Fig. 2(a)), or a charge-density wave (CDW) along the DW. This extra periodicity is responsible for the new structures in the structure factor $S(k)$ at $\boldsymbol{k}=( \pm \pi / 4 a, \pi / 2 a)$ (see Fig. 2(b)). Such structures are much weaker than those at $\boldsymbol{k}=( \pm 3 \pi / 4 a, 0)$ and $( \pm 3 \pi / 4 a, \pi)$ which originate from the stripe solid, but should in principle be observable. Thus, our qualitative prediction for stability of the HFDWs can be tested experimentally. 
(a)

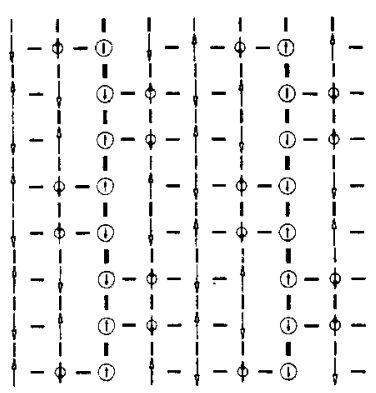

(b)

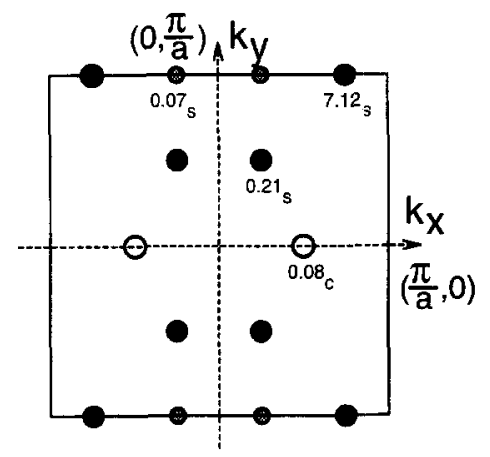

Fig. 2. (a) The $(0,1)$ striped phase with two HFDWs with on-wall SDW, as obtained for $V=0, i=0.5$, and $U / t \simeq 4.5$. The size of the circles and the thickness of the bonds indicate the excess bole density, and the bond contractions, respectively (normalized with regard to the largest/smallest bond-lengths, occurring along/perpendicular to the wall). (b) The same structure in reciprocal space, showing the spin ( $s$, closed circle) and charge (c, open circle) harmonics and their relative strength in $S(k)$.

The stability of the HFDWs can be also understood from the band structure shown in Fig. 3. The mid-gap states are here only quarter filled, and the gap opens at $k_{y}=\pi / 4$. Thus, the system with HFDWs is insulating due to the quadrupling of the unit cell along the wall. We note that part of the binding energy of a FDW gets lost due to the partial filling of the mid-gap soliton states, but is opposed by certain energy gain due to a weak binding in the direction along the wall.

If these new harmonics are indeed observed, the question arises why the HFDWs are energetically unfavourable in semiclassical calculations. We note that there is no particular reason for stability of the FDWs, as the gap between the top of the bulk bands

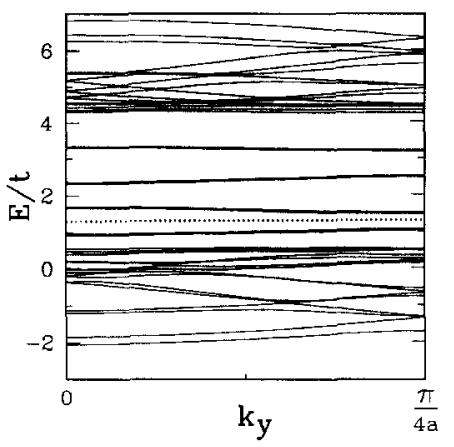

Fig. 3. The band structure as a function of parallel momentum $k_{y}$ for the HFDW of SDW type (Fig. 2), obtained for a $9 \times 2$ unit cell with $U / t=5$, and $\lambda=V=0$. Note that a sizable gap opens up at the Fermi-energy (dashed line) and the mid-gap bands are nearly dispersionless.

and the bottom of the soliton mid-gap band does not follow from an extra periodicity, unlike the one for the HFDWs. Thus, one might expect that some renormalization of parameters, further extension of the single-band model (1), or an explicit treatment of a multiband one, might give a different result on a semiclassical level. If not, one is left with quantum fluctuations which are certainly different for two kinds of the walls, but they are non-Gaussian and thus not easy to treat [7]. But a better understanding of a stripe solid in the cuprates at $\delta=\frac{1}{8}$ doping and of its melting into the stripe liquid is of central importance for a quantitative description of transport in the cuprates, and of the microscopic mechanism of superconductivity itself.

We acknowledge the partial support by the Committee of Scientific Research (KBN) of Poland, Project 2 P03B 144 08, and by Dutch Royal Academy of Sciences (KNAW).

\section{References}

[1] J. Zaanen and O. Gunnarson, Phys. Rev. B 40 (1989) 7391.

[2] D. Poilblanc and T.M. Rice, Phys. Rev. B 39 (1989) 9749; M. Inui and P.B. Littlewood, Phys. Rev. B 44 (1991) 4415.

[3] J. Zaanen and P.B. Littlewood, Phys. Rev. B 50 (1994) 7222.

[4] J.M. Tranquada et al., Phys. Rev. Lett. 73 (1994) 1003; J.M. Tranquada et al., Phys. Rev. B 52 (1995) 3581.

[5] C.H. Chen and S.-W. Cheong, Phys. Rev. Lett. 76 (1996) 4042. 
[6] H. Viertiö and T.M. Rice, J. Phys.: Condens. Matter 6 (1994) 7091.

[7] J. Zaanen, M. Horbach and W. van Saarloos, Phys. Rev. B 53 (1996) 8671; H. Eskes et al., Phys. Rev. B 54 (1996).
[8] J.M. Tranquada et al., Nature 375 (1995) 561.

[9] L.F. Feiner, J.H. Jefferson and R. Raimondi, Phys. Rev. B 53 (1996) 8751; R. Raimondi, J.H. Jefferson and L.F. Feiner, Phys. Rev. B 53 (1996) 8774.

[10] J. Zaanen and A.M. Oleś, Ann. Physik 5 (1996) 224. 